\title{
Purinergic signaling in the gastrointestinal tract
}

\author{
James J. Galligan
}

Published online: 29 May 2008

(C) Springer Science + Business Media B.V. 2008

The gastrointestinal (GI) tract (including the pancreas) and its blood supply are controlled by nerves and hormonal and paracrine signaling molecules. The nerves supplying the gut include the parasympathetic and sympathetic divisions of the autonomic nervous system and the enteric nervous system (ENS). The ENS is the division of the autonomic nervous system that is intrinsic to the GI tract. The GI tract is also supplied by vagal sensory afferent and spinal sensory afferent nerves. There are many neurotransmitters that can be released from the autonomic nerves supplying the gut; however, data from recent studies indicate that purines are important neurotransmitters that contribute to control of GI motility, secretion, blood flow, sensation, and immune function in the gut. The following supplement contains five chapters that summarize and analyze recent data highlighting the importance of purinergic mechanisms controlling gut function in health and disease.

ATP acting at P2X and P2Y receptors is an important excitatory neurotransmitter in enteric ganglia. The myenteric and submucosal plexuses contain synapses where ATP has been shown to contribute to synaptic excitation. The ENS is one of only a few parts of the nervous system where ATP mediates synaptic responses in functionally identified nerve pathways [1]. The review provided by Drs. Paul Bertrand and Jianhua Ren (Chapter 1: "Purinergic receptors and synaptic transmission in enteric neurons") presents an analysis and discussion of recent data supporting a role for ATP as a synaptic transmitter in the myenteric and

\footnotetext{
J. J. Galligan $(\triangle)$

Department of Pharmacology and Toxicology and the

Neuroscience Program, Michigan State University,

Life Science B328,

East Lansing, MI 48824, USA

e-mail: galliga1@msu.edu
}

submucosal plexuses. Bertrand and Ren discuss the contributions of ATP to fast and slow synaptic excitation in enteric ganglia, and they also review recent evidence relevant to the molecular identity of the purinergic receptors mediating synaptic excitation. Finally, the authors speculate about the possible role of purinergic neurotransmission in enteric ganglia in motility or secretory disorders in the gut.

Neurotransmission in enteric ganglia controls gastrointestinal motility, and purinergic signaling between neurons and between neurons and smooth muscle is an important component of the mechanisms controlling propulsive motility patterns. In the second chapter ("Purinergic mechanisms in the control of gastrointestinal motility"), Dr. Joel Bornstein reviews the recent literature supporting a contribution of purinergic signaling to integrative control of gut motility. Specifically, Bornstein discusses the role of purinergic transmission in each component of reflex pathways. This includes the role of purinergic signaling in reflex initiation and sensory transduction, integration of the sensory signal in enteric ganglia and purineric transmission to the muscle layers. Purinergic transmission facilitates coordinated contraction and relaxation of the muscle layers.

Just as purinergic neurotransmission in the myenteric plexus and to the muscle layers is important for normal propulsive motility patterns, purinergic neurotransmission is an important component of secretomotor control. In addition, ATP and other purines can contribute to secretomotor control via paracrine signaling mechanisms from non-neuronal cells types, including enterochromaffin cells. In Chapter 3, Dr. Fivos Christofi provides an extensive review ("Purinergic receptors and gastrointestinal secretomotor function") of purinergic mechanisms controlling intestinal secretion, particularly electrogenic chloride secretion.

The splanchnic circulation is a critical vascular bed in that it receives a large fraction of cardiac output. Therefore, 
blood flow through the splanchnic bed will have an important impact on blood pressure control and overall hemodynamics. In addition, control of blood flow through splanchnic blood vessels is important for gastrointestinal function and absorption of nutrients postprandially. Splanchnic blood vessels are controlled largely by the sympathetic nervous system, and ATP is a co-transmitter released with norepinephrine from perivascular nerves. Purines released from non-neuronal cells can also act locally in the splanchnic circulation to alter vascular tone. Dr. Antonio Albino-Teixeira (Chapter 4: "Purinergic receptors in the splanchnic circulation") provides a review and analysis of the literature related to purinergic mechanisms controlling vascular tone in the splanchnic circulation. Albino-Teixeira also discusses purinergic signaling and pathophysiological changes in splanchnic blood flow.

Finally, Dr. Ivana Novak discusses purinergic contributions to the control of pancreatic function (Chapter 5: "Purinergic receptors and pancreatic exocrine and endocrine function"). Pancreatic function is controlled partly by the autonomic nervous system as it is supplied by sympathetic and parasympathetic nerve fibers. ATP and other purines may contribute to neurotransmission from these extrinsic pancreatic nerves. Numerous cell types are present in the pancreas and each of these cell types has the potential to use purines as intercellular signaling molecules. This is important because purines can then modulate the endocrine and exocrine functions of the pancreas.

Two important aspects of purinergic signaling in the gut not covered here are related to purines and sensory nerve function and purines in gut immune-system function and inflammation. Purinergic signaling mechanisms in the gut wall lead to nociceptive sensation, and these mechanisms can be upregulated in animal models of visceral hypersensitivity [2]. There is an extensive literature on purinergic contributions to pain sensation in the gut and elsewhere, and there are a number of excellent recent reviews that have covered this topic in detail [3-7]. Intestinal inflammation is often part of the pathophysiology of visceral pain and hypersensitivity $[6,7]$, but inflammation can also lead to changes in gastrointestinal motor and secretory function $[8$, $9,10]$. Purinergic signaling is an important part of the inflammatory response in the gastrointestinal tract, and this topic has been reviewed recently [11].

\section{References}

1. North RA (2002) Molecular physiology of P2X receptors. Physiol Rev 82:1013-1067

2. Xu GY, Shenoy M, Winston JH, Mittal S, Pasricha PJ (2008) P2X receptor-mediated visceral hyperalgesia in a rat model of chronic visceral hypersensitivity. Gut. DOI 10.1136/gut.2007.134221

3. Burnstock G (2007) Physiology and pathophysiology of purinergic neurotransmission. Physiol Rev 87:659-797

4. Khakh BS, North RA (2006) P2X receptors as cell-surface ATP sensors in health and disease. Nature 442:527-532

5. Blackshaw LA, Brookes SJ, Grundy D, Schemann M (2007) Sensory transmission in the gastrointestinal tract. Neurogastroenterol Motil 19(1 Suppl):1-19

6. Vergnolle N (2008) Postinflammatory visceral sensitivity and pain mechanisms. Neurogastroenterol Motil 20(Suppl 1):73-80

7. Wirkner K, Sperlagh B, Illes P (2007) P2X $\mathrm{X}_{3}$ receptor involvement in pain states. Mol Neurobiol 36:165-183

8. Holzer P (2007) Role of visceral afferent neurons in mucosal inflammation and defense. Curr Opin Pharmacol 7:563-569

9. Chandrasekharan B, Srinivasan S (2007) Diabetes and the enteric nervous system. Neurogastroenterol Motil 19:951-960

10. Ohama T, Hori M, Ozaki H (2007) Mechanism of abnormal intestinal motility in inflammatory bowel disease: how smooth muscle contraction is reduced. J Smooth Muscle Res 43:43-54

11. Kolachala VL, Bajaj R, Chalasani M, Sitaraman SV (2008) Purinergic receptors in gastrointestinal inflammation. Am J Physiol 294:G401-410 